\title{
Guidelines for Acute Ischemic Stroke Treatment
}

\author{
Shuwei Qiu' ${ }^{1}$ Yun $\mathrm{Xu}^{1,2,3,4}$
}

Received: 2 February 2020/ Accepted: 18 March 2020/Published online: 19 June 2020

(C) Shanghai Institutes for Biological Sciences, CAS 2020

Acute ischemic stroke (AIS) is one of the serious diseases endangering human health worldwide; it usually leads to serious handicap with a high risk of recurrence and death. Despite the global burden of stroke, great advances are still being made. Since the publication of the 2013 guidelines for the early management of AIS patients, new high-quality evidence has resulted in prominent changes in the evidence-based treatment of AIS, such as image assessment, treatment beyond the time-window, new thrombolytic drugs, thrombolysis for strokes with an unknown onset time, and dual antiplatelet therapy for minor strokes. Especially in 2015, publication of the results of five clinical trials on mechanical thrombectomy contributed to the focused update of the 2013 guidelines for the early management of patients with AIS regarding endovascular treatment. Five years later, the publication of the DAWN and DEFUSE 3 results focusing on AIS beyond $6 \mathrm{~h}$ from onset demonstrated a significant benefit of mechanical thrombectomy for selected stroke patients $6 \mathrm{~h}-24 \mathrm{~h}$ from onset. Consequently, the American Heart Association/ American Stroke Association (AHA/ASA) issued new

Yun Xu

xuyun20042001@aliyun.com

1 Department of Neurology, Drum Tower Hospital, Medical School and The State Key Laboratory of Pharmaceutical Biotechnology, Institute of Brain Science, Nanjing University, Nanjing 210008, China

2 Jiangsu Key Laboratory for Molecular Medicine, Medical School of Nanjing University, Nanjing 210008, China

3 Jiangsu Province Stroke Center for Diagnosis and Therapy, Nanjing 210008, China

4 Nanjing Medical Center for Neurology, Nanjing 210008, China guidelines to supersede the 2013 guidelines in January 2018 [1], of which certain chapters were unfortunately deleted due to controversy. Here, we highlight some of the new recommendations and comment on imaging evaluation, intravenous (IV) thrombolysis, mechanical thrombectomy, and antiplatelet treatment from the AHA/ASA and the China 2018-2019 AIS guidelines [2, 3].

\section{Precision Thrombolysis and Thrombectomy under Multimodal Imaging Guidance}

The benefit of IV alteplase is time-dependent, and earlier treatment within the therapeutic window leads to a greater benefit. Studies have shown that median or mean door-toimaging times of $\leq 20 \mathrm{~min}$ can be achieved in a variety of different hospital settings. As a result, the 2018 guidelines recommended that "brain imaging studies can be performed within 20 minutes of arrival in the emergency department (ED) in at least $50 \%$ of patients who may be candidates for IV alteplase and/or mechanical thrombectomy". However, the absence of detailed screening information in the DAWN and DEFUSE 3 trials limits estimation of the true impact of time on prognosis in the population beyond the 6-h time window, leading to the deletion of these specific values from the 2019 update. Nevertheless, brain imaging should be performed as quickly as possible and these specific values could be set as potential targets. Moreover, the new guidelines emphasize that, in patients eligible for IV alteplase, treatment should be initiated as quickly as possible and not delayed for additional multimodal neuroimaging, including enhanced computed tomography/magnetic resonance imaging (CT/MRI), CT angiography (CTA) with CT perfusion imaging (CTP) or MR angiography with diffusion- 
weighted imaging (DWI) or MRI perfusion imaging. Noncontrast CT was the only neuroimaging modality used in the NINDS and ECASS III trials and it is therefore sufficient for decisions about IV alteplase in most patients. These recommendations imply that suspected patients eligible for thrombolysis should receive non-contrast CT scanning rather than multimodal neuroimaging before IV alteplase treatment, even if mechanical thrombectomy is being considered.

For certain stroke patients, some new recommendations for brain and vessel imaging have been made for eligibility evaluation of IV thrombolysis or mechanical thrombectomy, owing to the favorable results of up-to-date clinical trials. With respect to wake-up stroke or an unknown onset time $>4.5 \mathrm{~h}$, the guidelines recommend that a DWI-FLAIR (fluid attenuation inversion recovery) mismatch can be useful for selecting those who can benefit from IV alteplase treatment within $4.5 \mathrm{~h}$ of stroke symptom recognition by virtue of the WAKE-UP trials [4]. Regarding stroke in the anterior circulation $6 \mathrm{~h}-24 \mathrm{~h}$ from last time seen normal, the guidelines recommend that CTP or diffusion-weighted MRI with or without MRI perfusion is able to aid in patient selection for mechanical thrombectomy according to the DAWN and DEFUSE 3 clinical trials [5, 6]. In fact, multimodal CT is more widely available, faster, and more convenient than multimodal MRI. Therefore, multimodal CT is more widely used in China.

Besides, the guidelines do not recommend obtaining an MRI to exclude cerebral microbleeds before IV alteplase administration for eligible patients, and also recommend that for patients with suspected intracranial large vessel occlusion and no history of renal impairment, it is reasonable to proceed with CTA before obtaining a serum creatinine concentration for selection of endovascular treatment. The aim of these detailed recommendations is to decrease the door-to-needle time and prevent delay of thrombolysis, and furthermore, standardization of these evaluations is beneficial for the prevention of controversy and conflicts in clinical practice.

\section{Thrombolysis}

The safety and efficacy of IV thrombolysis within the first $3 \mathrm{~h}$ after stroke onset are solidly supported by combined data from multiple randomized controlled trials (RCTs) and have been confirmed by extensive community experience in many countries. The eligibility criteria for IV alteplase have evolved over time as its usefulness and the true risks have become clearer. Therefore, in contrast to the 2013 guidelines, the 2018 guidelines and 2019 update specify and revise the indications and contraindications of
IV thrombolysis, which guarantees that more AIS patients benefit from this treatment.

For patients $>80$ years old presenting within the 3 -h to 4.5-h window, the 2019 update recommends that IV alteplase is safe and can be as effective as in younger patients, unlike the 2013 guidelines. The ECASS III trial specifically confirmed the efficacy of IV alteplase in the time period from $3 \mathrm{~h}$ to $4.5 \mathrm{~h}$ after symptom onset; however it excluded patients $>80$ years old. Later, pooled analysis of multiple trials indicated that patients older than 80 years achieved benefits similar to those aged 80 years or younger, particularly when treated early [7].

In the 2019 update, mild stroke is set as a specific subitem, and three new recommendations are provided. For mild disabling stroke symptoms, IV thrombolysis may be reasonable for patients who can be treated within $3 \mathrm{~h}-4.5 \mathrm{~h}$ of ischemic stroke onset or patients last time known well. However, it should be noted that for patients with mild non-disabling stroke symptoms [NIHSS (NIH Stroke Scale) score 0-5], IV alteplase is not recommended for those who can be treated within $3 \mathrm{~h}$ or within $3 \mathrm{~h}-4.5 \mathrm{~h}$ of symptom onset or patient last time known well. This is based on the PRISMS RCT results, which demonstrated that there was no benefit of IV alteplase in patients with mild (NIHSS score 0-5) AIS within $3 \mathrm{~h}$ of onset [8]. However, due to the very early termination of this study, additional research may be warranted.

AIS patients with an unknown time of onset are recommended to receive IV alteplase treatment within $4.5 \mathrm{~h}$ of stroke symptom recognition guided by a mismatch between diffusion-weighted MRI and FLAIR in the region of ischemia. The evidence came from the WAKE-UP RCT in 2018 which randomized 503 AIS patients who awoke with stroke or had an unclear time of onset and could be treated with IV alteplase within $4.5 \mathrm{~h}$ of stroke symptom recognition [4]. This trial demonstrated that the primary end-point of a modified Rankin scale ( $\mathrm{mRS}$ ) score of $0-1$ at 90 days was achieved in $53.3 \%$ of the alteplase group and in $41.8 \%$ of the placebo group $(P=0.02)$. Later, a metaanalysis demonstrated that AIS patients $4.5 \mathrm{~h}-9 \mathrm{~h}$ from stroke onset or wake-up stroke with salvageable brain tissue, assessed by perfusion-weighted MRI (PW-MRI) or CTP, who were treated with alteplase achieved better functional outcomes at 3 months than did patients given placebo (OR 1.86, 95\% CI 1.15-2.99, $P=0.011$ )[9]. The rate of symptomatic intracerebral hemorrhage was higher with alteplase, but this increase did not negate the overall net benefit of thrombolysis. This means that the timewindow of IV thrombolysis for AIS is extended for eligible stroke patients selected by PW-MRI or CTP, meaning more patients benefit from this treatment.

Besides, these guidelines give new recommendations on some particular conditions. First, for stroke patients with a 
hyperdense MCA sign, IV alteplase treatment can be beneficial, because analyses of data from RCTs of IV alteplase for AIS have shown no statistically significant deleterious interaction on clinical outcomes between alteplase treatment and the hyperdense MCA sign on baseline CT. Second, IV alteplase can be beneficial for adults with known sickle-cell disease presenting with an AIS. Third, for eligible patients with previous cerebral microbleeds demonstrated on MRI, administration of IV alteplase is reasonable with $\leq 10$ microbleeds while IV thrombolysis may increase the risk of symptomatic intracerebral hemorrhage with $>10$ microbleeds unless there is potential for substantial benefit. All these new recommendations suggest that thrombolysis is beneficial for specific conditions, ensuring that more patients are treated with alteplase.

\section{Mechanical Thrombectomy}

Recently, a major advance was achieved in the endovascular treatment of early stroke with an extended time window. Due to the results of the DAWN and DEFUSE 3 trials, two new recommendations related to mechanical thrombectomy were provided for symptom onset $>6 \mathrm{~h}$ in the 2018 guidelines and a more elaborate format rearrangement is made in the 2019 update, leading to the selection of more eligible patients for endovascular treatment through extension of the time window.

In the 2015 endovascular guidelines, the results from 6 randomized trials (MR CLEAN, SWIFT PRIME, EXTEND-IA, ESCAPE, REVASCAT, and THRACE) supported the Class I, LOE (level of evidence) A recommendations of mechanical thrombectomy for a defined group of patients within $6 \mathrm{~h}$ of stroke onset, predominantly using stent retriever devices. In 2018, two outstanding trials (DAWN and DEFUSE 3) demonstrated the efficacy and safety of thrombectomy for stroke patients $>6 \mathrm{~h}$ after onset. The DAWN trial used clinical imaging mismatch (a combination of NIHSS score and imaging findings on CTP or diffusion-weighted MRI) as eligibility criteria to select patients with large anterior circulation vessel occlusion for treatment with mechanical thrombectomy between $6 \mathrm{~h}$ and $24 \mathrm{~h}$ from last time known normal. This trial demonstrated an overall benefit in functional outcome at 90 days in the treatment group [5] (mRS score $0-2,49 \%$ versus 13\%; adjusted difference, 33\%; 95\% CI, 21-44). The DEFUSE 3 trial used perfusion-core mismatch and maximum core size as imaging criteria to select patients with large anterior circulation occlusion $6 \mathrm{~h}-16 \mathrm{~h}$ from last time seen well for mechanical thrombectomy. This trial showed a benefit in functional outcome at 90 days in the treated group[6] (mRS score $0-2,44.6 \%$ versus $16.7 \%$; relative risk, 2.67 ; $95 \%$ CI,
1.60-4.48; $P<0.0001)$. DAWN and DEFUSE 3 are the only RCTs showing a benefit of mechanical thrombectomy $>6 \mathrm{~h}$ from onset. Therefore, only the eligibility criteria from one or the other of these trials should be used for patient selection. Although future RCTs may demonstrate that additional eligibility criteria can be used to select patients who benefit from mechanical thrombectomy, at this time, the DAWN or DEFUSE-3 eligibility should be strictly adhered to in clinical practice.

\section{Antiplatelet Treatment}

Antiplatelet treatment plays a fundamental role in AIS treatment and prevention. The new evidence mainly comes from the results of clinical trials in patients with minor stroke and high-risk transient ischemic attack (TIA). First of all, one modification of the new guidelines is related to the dual antiplatelet therapy of minor non-cardioembolic ischemic stroke (NIHSS score $\leq 3$ ), with the recommendation strength elevated from COR (class of recommendation) IIa, LOE B-randomized to COR I, LOE A by virtue of evidence from the CHANCE and POINT trials. In 2013, the CHANCE trial conducted in China studied the efficacy of short-term dual antiplatelet therapy begun within $24 \mathrm{~h}$ in patients with minor stroke or high-risk TIA (ABCD2 score $\geq 4$ ). The dosage regimen was clopidogrel at an initial dose of $300 \mathrm{mg}$, followed by $75 \mathrm{mg}$ per day for 90 days, plus aspirin at $75 \mathrm{mg}$ per day for the first 21 days or to placebo plus aspirin ( $75 \mathrm{mg}$ per day for 90 days). All participants received open-label aspirin at a clinician-determined dose of $75 \mathrm{mg}-300 \mathrm{mg}$ on day 1 . The primary outcome of recurrent stroke (ischemic or hemorrhagic) at 90 days favored dual antiplatelet therapy over aspirin alone [10] (HR 0.68; 95\% CI, 0.57-0.81; $P<0.001$ ). In 2018, the POINT trial conducted in western countries enrolled a similar population of patients with minor stroke (NIHSS score $\leq 3$ ) or high-risk TIA (ABCD2 score $\geq 4$ ) within $12 \mathrm{~h}$ of symptom onset. Patients were randomized to either clopidogrel plus aspirin (600 mg loading dose of clopidogrel followed by $75 \mathrm{mg}$ /day from days 2-90) plus openlabel aspirin (50 mg/day-325 mg/day) versus aspirin alone (50 mg/day-325 mg/day) for 90 days. Compared with aspirin alone, aspirin plus clopidogrel resulted in fewer ischemic events (5\% versus 6.5\%; HR, 0.75 [95\% CI, 0.59-0.95]; $P=0.02$ ) but more major hemorrhages[11] (0.9\% versus $0.4 \%$; HR, 2.32 [95\% CI, 1.10-4.87]; $P=$ 0.02). A pooled analysis including FASTER, CHANCE, and POINT concluded that discontinuation of dual antiplatelet therapy within 21 days of initiation, and possibly as early as 10 days, is likely to maximize benefit and minimize harm [12]. 
Another new recommendation related to antiplatelet treatment is that ticagrelor is not recommended over aspirin for the treatment of patients with minor acute stroke based on the SOCRATES trial [13] [ticagrelor versus aspirin begun within $24 \mathrm{~h}$ in patients with minor stroke (NIHSS score $\leq 5$ ) or TIA (ABCD2 score $\geq 4)]$. With a primary outcome of time to the composite end-point of stroke, myocardial infarction, or death up to 90 days, ticagrelor was not found to be superior to aspirin (HR, 0.89 [95\% CI, $0.78-1.01] ; P=0.07)$. However, because there were no significant safety differences between the two groups, ticagrelor may be a reasonable alternative in stroke patients who have a contraindication for aspirin.

One minor revision of the 2018 guidelines is that the specific dosage recommendation, "initial dose is $325 \mathrm{mg}$ " was removed, because the safety and benefit of antiplatelet treatment with aspirin for AIS patients were established by two large clinical trials administering doses between 160 $\mathrm{mg}$ and $300 \mathrm{mg}$ within $24 \mathrm{~h}-48 \mathrm{~h}$ after onset [2].

In conclusion, increasing evidence from high-quantity clinical trials provides new support for more detailed recommendations for the early management of AIS and eventually provides more potential benefit for patients.

Acknowledgements This insight article was supported by the National Natural Science Foundation of China (81920108017, 81630028, and 81870928), the Key Research and Development Program of Jiangsu Province, China (BE2016610), and Jiangsu Province Key Medical Discipline (ZDXKA2016020).

Conflict of interest The authors claim that there are no conflicts of interest.

\section{References}

1. Powers WJ, Rabinstein AA, Ackerson T, Adeoye OM, Bambakidis NC, Becker K, et al. 2018 Guidelines for the Early Management of Patients With Acute Ischemic Stroke: A guideline for healthcare professionals from the American Heart Association/American Stroke Association. Stroke 2018, 49: e46-e110.
2. Powers WJ, Rabinstein AA, Ackerson T, Adeoye OM, Bambakidis NC, Becker K, et al. Guidelines for the Early Management of Patients With Acute Ischemic Stroke: 2019 Update to the 2018 Guidelines for the Early Management of Acute Ischemic Stroke: A guideline for healthcare professionals from the American Heart Association/American Stroke Association. Stroke 2019, 50: e344-e418.

3. Chinese Society of Neurology and Chinese Stroke Society. Chinese guidelines for diagnosis and treatment of acute ischemic stroke 2018. Chin J Neurol, 2018. 51: 666-682.

4. Thomalla G, Simonsen CZ, Boutitie F, Andersen G, Berthezene Y, Cheng B, et al. MRI-guided thrombolysis for stroke with unknown time of onset. N Engl J Med 2018, 379: 611-622.

5. Nogueira RG, Jadhav AP, Haussen DC, Bonafe A, Budzik RF, Bhuva P, et al. Thrombectomy 6 to 24 hours after stroke with a mismatch between deficit and infarct. N Engl J Med 2018, 378: $11-21$.

6. Albers GW, Marks MP, Kemp S, Christensen S, Tsai JP, OrtegaGutierrez S, et al. Thrombectomy for stroke at 6 to 16 hours with selection by perfusion imaging. N Engl J Med 2018, 378: 708-718.

7. Wardlaw JM, Murray V, Berge E, del Zoppo G, Sandercock P, Lindley RL, et al. Recombinant tissue plasminogen activator for acute ischaemic stroke: an updated systematic review and metaanalysis. Lancet 2012, 379: 2364-2372.

8. Khatri P, Kleindorfer DO, Devlin T, Sawyer RN, Jr., Starr M, Mejilla $\mathrm{J}$, et al. Effect of alteplase vs aspirin on functional outcome for patients with acute ischemic stroke and minor nondisabling neurologic deficits: The PRISMS randomized clinical trial. JAMA 2018, 320: 156-166.

9. Campbell BCV, Ma H, Ringleb PA, Parsons MW, Churilov L, Bendszus $\mathrm{M}$, et al. Extending thrombolysis to $4.5-9 \mathrm{~h}$ and wakeup stroke using perfusion imaging: a systematic review and metaanalysis of individual patient data. Lancet 2019, 394: 139-147.

10. Wang Y, Zhao X, Liu L, Wang D, Wang C, Li H, et al. Clopidogrel with aspirin in acute minor stroke or transient ischemic attack. N Engl J Med 2013, 369: 11-19.

11. Johnston SC, Easton JD, Farrant M, Barsan W, Conwit RA, Elm $\mathrm{JJ}$, et al. Clopidogrel and aspirin in acute ischemic stroke and high-risk TIA. N Engl J Med 2018, 379: 215-225.

12. Hao Q, Tampi M, O'Donnell M, Foroutan F, Siemieniuk RA, Guyatt G. Clopidogrel plus aspirin versus aspirin alone for acute minor ischaemic stroke or high risk transient ischaemic attack: systematic review and meta-analysis. BMJ 2018, 363: k5108.

13. Johnston SC, Amarenco P. Ticagrelor versus aspirin in acute stroke or transient ischemic attack. N Engl J Med 2016, 375: 1395. 